ВЗАИМОСВЯЗЬ ОЖИРЕНИЯ И РАКА ПРЕДСТАТЕЛЬНОЙ ЖЕЛЕЗЫ (ОБЗОР)

(с) М.Н. Пешков ${ }^{1 *}$, Г.П. Пешкова², И.В. Решетов ${ }^{3}$

'Федеральный научно-клинический центр специализированных видов медицинской помощи и медицинских технологий Федерального Медико-биологического агентства, Москва, Россия

Рязанский государственный медицинский университет имени академика И.П. Павлова, Рязань, Россия

${ }^{3}$ Первый Московский государственный медицинский университет им. И.М. Сеченова (Сеченовский Университет),

Москва, Россия

Ожирение является критическим фактором риска развития рака предстательной железы (РПЖ). Жировая ткань играет важную роль в развитии опухоли, включая рост, инвазию и метастазирование. Диета и диетические компоненты влияют на прогрессирование РПж; однако механизмы, лежащие в основе этих ассоциаций, остаются неясными. Опухолевые клетки предстательной железы с экстрапростатическим распространением образуют новое микроокружение в перипростатической жировой ткани, которое изменяет характер этих взаимодействий и способствует прогрессированию опухоли.

Гиперинсулинемия приводит к повышению уровня свободного или биологически активного инсулиноподобного фактора роста (IGF-1) из-за снижения выработки IGF-связывающих белков. Гипоандрогенизм способствует развитию более агрессивного типа опухоли РПЖ (более высокие показатели Глисона). Адипокины жировой ткани и цитокины (например, интерлейкин-6 (IL-6) и фактор некроза опухоли (TNF-a)), ангиогенные факторы (например, фактор роста эндотелия сосудов (VEGF), апелин (AGTRL1)) и другие факторы (например, лептин и адипонектин) оказывают множественное влияние на клетки рака предстательной железы. Опухолевые клетки напрямую или косвенно взаимодействуют с адипоцитами.

Желтый (недеятельный) костный мозг - это жировая ткань с отдельными островками ретикулярной ткани. Он находится в костномозговых каналах трубчатых костей и в частях ячеек губчатого вещества костей. Костная ткань является объектом наиболее частого метастазирования при раке простаты, и с возрастом содержание жировых клеток в ней увеличивается. Жировая ткань костного мозга взаимодействует с опухолевыми клетками, остеобластами и другими стромальными клетками и участвует в организации микроокружения опухоли. Адипокины являются ключевыми молекулами взаимодействия между опухолевыми клетками и жировой тканью, которое осуществляется при помощи разных механизмов. Более глубокое понимание роли жировой ткани в индукции и прогрессировании РПЖ позволит выработать эффективные терапевтические стратегии при данном заболевании.

КЛЮЧЕВЫЕ СЛОВА: ожирение; индекс массы тела (ИМТ); адипоциты; адипокины; инсулиноподобный фактор роста (ИФР-1); тестостерон; рак предстательной железы.

\title{
THE RELATIONSHIP OF OBESITY AND PROSTATE CANCER (REVIEW)
}

\author{
(c) Maxim N. Peshkov*, Galina P. Peshkova², Igor V. Reshetov ${ }^{3}$
}

${ }^{1}$ Federal Research and Clinical Center of Specialized Medical Care and Medical Technologies FMBA of Russia, Moscow, Russia; 2I.P. Pavlov Ryazan State Medical University, Ryazan, Russia;

3..M. Sechenov First Moscow State Medical University (Sechenov University), Moscow, Russia

Obesity is a critical risk factor for prostate cancer (PCa). Adipose tissue plays an important role in tumor development, including growth, invasion, and metastasis. Diet and dietary components affect the progression of prostate cancer; however, the mechanisms underlying these associations remain unclear. Extraprostatic prostate tumor cells form a new microenvironment in the periprostatic adipose tissue, which alters these interactions and promotes tumor progression.

Hyperinsulinemia leads to an increase in the level of free or biologically active insulin-like growth factor (IGF-1) due to a decrease in the production of IGF-binding proteins. Hypoandrogenism promotes the development of a more aggressive type of prostate cancer (higher Gleason scores). Adipokines of adipose tissue and cytokines (for example, interleukin-6 (IL-6) and tumor necrosis factor (TNF-a), angiogenic factors (for example, vascular endothelial growth factor (VEGF), apelin (AGTRL1) and other factors (for example, leptin and adiponectin) have multiple effects on prostate cancer cells. Tumor cells interact directly or indirectly with adipocytes.

Yellow (inactive) bone marrow is adipose tissue with separate islands of reticular tissue. It is located in the medullary canals of the tubular bones and in parts of the cells of the cancellous bone. Bone tissue is the object of the most frequent metastasis in prostate cancer, and with age, the content of fat cells in it increases. Bone marrow adipose tissue interacts with tumor cells, osteoblasts and other stromal cells and participates in the organization of the tumor microenvironment. Adipokines are key molecules in the interaction between tumor cells and adipose tissue, which is carried out through various mechanisms. A better understanding of the role of adipose tissue in the induction and progression of prostate cancer will lead to effective therapeutic strategies for this disease.

KEYWORDS: obesity; body mass index (BMI); adipocytes; adipokines; insulin-like growth factor (IGF-1); testosterone; prostate cancer. 


\section{ВВЕДЕНИЕ}

Рак предстательной железы (РПЖ) продолжает оставаться одним из самых распространенных онкологических заболеваний мужчин во всем мире. В 2018 г. в России выявлено 36725 новых случаев РПЖ, при этом на учете с данным диагнозом состояли 220264 пациентов [1].

По данным Национального института рака (NCl), прогноз на 2020 г. указывает на 191930 новых случаев рака простаты и 33330 смертей от этой болезни [2], при этом 5-летняя выживаемость при локализованном РПЖ составляет 98\%. РПЖ будет диагностирован почти у каждого пятого мужчины в США и примерно 3\% мужчин, которые, как ожидается, умрут от этой болезни [3]. Заболеваемость раком простаты в азиатских странах, включая Японию, намного ниже, чем в западных странах. Тем не менее заболеваемость раком простаты в азиатских странах неуклонно растет в течение последних нескольких десятилетий [4].

Экстрапростатическое распространение опухоли определяется как $\mathrm{T}_{3-4}$ в системе стадий опухоли (TNM), поражение лимфатических узлов, наличие метастазов при РПЖ, хорошо известно как связанное с неблагоприятным прогнозом. Стадирование опухолевого процесса является основополагающей функцией и должно быть точно определено для оптимального ведения пациента, в том числе и после проведения хирургического лечения - радикальной простатэктомии [5]. Простата окружена перипростатической жировой тканью. Включения опухолевых клеток в перипростатической жировой ткани являются наиболее распространенной находкой экстрапростатической инвазии [6].

Жировая ткань состоит в основном из адипоцитов, присутствуют дополнительные типы клеток, включая перициты, моноциты, макрофаги, лимфоциты, фибробласты, эндотелиальные клетки сосудов и плюрипотентные стволовые клетки. Ранее считалось, что жировая ткань играет ограниченную физиологическую роль, главным образом в накоплении энергии и защите от низких температур. В настоящее время жировая ткань считается активным эндокринным органом, секретирующим факторы роста, хемокины или провоспалительные молекулы, называемые «адипокинами», которые влияют на обмен веществ и состояние иммунной системы. Физиологически адипокины регулируют аппетит, липидный обмен, гомеостаз глюкозы, чувствительность к инсулину, ангиогенез, кровяное давление и воспалительные процессы. Ожирение определяется увеличением жировой массы в результате энергетического дисбаланса. Гипертрофия адипоцитов при ожирении вызывает дисфункцию жировой ткани и воспаление путем увеличения секреции провоспалительных адипокинов из адипоцитов. Изменения биологии адипоцитов, связанные с гипертрофией адипоцитов, влияют на системные органы. Эпидемиологически ожирение связано с риском развития многих видов опухолей, включая рак пищевода, желудка, колоректальный, желчного пузыря, поджелудочной железы, эндометрия, яичников и почек $[7,8]$. Кроме того, ожирение связано с прогрессированием многих видов рака, включая рак предстательной железы, молочной железы, эндометрия, почек, поджелудочной железы, пищевода и щитовидной железы [9]. Кроме того, взаимодействие между нор- мальными адипоцитами и опухолевыми клетками может осуществляться в начале раковой инвазии в жировую ткань [10]. Таким образом, все больше данных свидетельствуют о значительной роли жировой ткани в развитии нескольких видов опухолей.

Поскольку ожирение и РПЖ затрагивают значительную часть мужского населения, связь между ними имеет большое значение для общественного здравоохранения. Авторами данной статьи основное внимание уделяется определению основных молекулярно-генетических механизмов, также рассматривается влияние ожирения на малигнизацию ткани предстательной железы.

\section{Методология поиска первоисточников}

Для основного поиска источников использовали интернет-ресурс PubMed, также использовали базу данных ELIBRARY. Сайты издательств Springer и Elsiver использовались для доступа к полному тексту статей. Этапы и ключевые слова поиска: 1-й этап: obesity, definition of obesity, prostate cancer, definition of prostate cancer; 2-й этап: obesity pathogenesis, obesity pathophysiology, prostate cancer pathogenesis; 3-й этап: obesity diagnostics, prostate cancer diagnostics; body mass index (BMI); 4-й этап: insulin-like growth factor (IGF)-1, leptin, interleukin-6 (IL-6), adiponectin; 5-й этап: microecology of adipose tissue.

\section{ВЗАИМОСВЯЗЬ ОЖИРЕНИЯ И РАКА ПРЕДСТАТЕЛЬНОЙ ЖЕЛЕЗЫ}

В США 14\% всех случаев смерти от рака среди мужчин и 20\% всех случаев смерти от рака среди женщин связаны с таким фактором риска, как избыточный вес и ожирение [11]. Эпидемиологические исследования показывают, что связь между ожирением и заболеваемостью раком простаты не до конца изучена. Некоторые авторы сообщают об отсутствии связи между ожирением и РПЖ $[12,13]$. Другая группа исследований демонстрирует сильную прямую связь ожирения со смертностью от рака предстательной железы [14, 15]. Кроме того, ожирение связано с повышенным риском биохимического рецидива рака простаты после радикальной простатэктомии (РПЭ), независимо от возраста на момент постановки диагноза и патологических особенностей опухоли [12]. Некоторые исследования предполагают, что ожирение снижает риск локализованного неагрессивного РПЖ, при этом повышает риск агрессивного РПЖ [13]. Ожирение связано с наличием простатической интраэпителиальной неоплазии предстательной железы в доброкачественных образцах при негативной первичной биопсии и с высоким риском малигнизации ткани простаты при последующих биопсиях. Следовательно, ожирение следует рассматривать как фактор риска при активном динамическом клиническом наблюдении за данными пациентами после первичной негативной биопсии [14].

На сегодняшний день исследования $[15,16,17]$ свидетельствуют о прямой положительной сильной связи между ожирением и риском развития РПЖ. Относительные риски (ОР) в этих исследованиях носят постоянный характер: от 1,01 (95\% доверительный интервал [ДИ] 1,00-1,02) на 1 кг/м² увеличения ИМТ [17] до 1,05 (95\% ДИ 1,01-1,08)) [16] и 1,03 (95\% ДИ 1,00-1,07) [15] 
на 5 кг/м² прироста ИМТ. Результаты отдельных исследований, определяющих вклад в итоговый результат этого метаанализа, имеют отличия: одни сообщают об отсутствии связи между ожирением и РПЖ [18], другие свидетельствуют, что ожирение является фактором риска развития опухоли $[19,20]$. Эти отдельные исследования сильно различаются по мощности, таким образом, внося различный вклад в выводы метаанализа. В когортном исследовании Engeland A. и его коллег сообщается об относительном риске (ОР) 1,09 (95\% ДИ 1,04-1,15) для мужчин с ожирением по сравнению с мужчинами с нормальным весом [18], вклад соответствует приблизительно 50\% случаев РПЖ в двух из трех метаанализов $[14,15]$. В третьем метаанализе [17] сообщается об ОР 1,4 (95\% ДИ 1,09-1,81) для мужчин с ожирением при сравнении с мужчинами с нормальным весом. В данной работе более половины случаев РПЖ приходится на исследование, проведенное Andersson S.O. и его коллегами [20]. С этими двумя скандинавскими исследованиями, составляющими большинство случаев РПЖ, связывают основное прямое положительное влияние ожирения на развитие опухоли.

При изучении географии заболеваемости, позволяющей разделить исследования Северной Америки от работ европейских коллег, возникает отчетливая картина. Среди исследований из Северной Америки не наблюдается влияния ожирения на риск развития РПЖ (OР 1,00; 95\% ДИ 0,96-1,03), в то время как в европейских и австралийских исследованиях наблюдается прямая сильная положительная связь ожирения с риском развития РПЖ (OР 1,04; 95\% ДИ 1,01-1,07) на 5 кг/м² прироста ИМТ [13]. Одно из возможных объяснений этих географических различий относится к различным показателям скрининга простатспецифического антигена (ПСА). В Европе, где скрининг на ПСА не так распространен, как в Соединенных Штатах, многие виды рака диагностируются на более поздних стадиях. В Соединенных Штатах оппортунистический скрининг ПСА широко распространен. Ранняя диагностика РПЖ возможна при выполнении биопсии простаты в раннем периоде у пациентов с повышенным уровнем ПСА. Это позволяет идентифицировать опухоль на ранней стадии и верифицировать ее с более низким прогностическим риском [21].

У пациентов с избыточной массой тела отмечается снижение уровня ПСА крови из-за гемодилюции [22]. Повышение уровня ПСА крови является показанием для проведения пункционной биопсии простаты. В странах с распространенным оппортунистическим скринингом ПСА, у пациентов с ожирением вероятность выполнения биопсии меньше, чем у мужчин с нормальным весом. Данное явление приводит к выявлению меньшего числа случаев рака на ранней стадии у людей с избыточным весом. Диагностическое «смещение» ослабляет силу прямой корреляционной связи между ожирением и риском РПж (т.е. ожирение «маскирует» появление опухоли в начале развития), это показывают многочисленные крупные проспективные когортные исследования в Соединенных Штатах с объемом выборки 400 000 пациентов [19, 20, 23]. Можно предположить взаимосвязь ожирения с распространенными формами заболеваний, при условии, что ожирение биологически связано с агрессивной формой рака простаты. Результаты проспективных исследований, изучающих взаимосвязь между ИМТ и риском смерти от РПЖ, неоднозначны. Так, в работах Wright M.Е. и его коллег, а также в исследовании Burton A. с коллегами наблюдается нулевая связь с летальным исходом от РПЖ $[24,25]$. В то же время в исследовании Okasha M. и его коллег наблюдается незначимая прямая связь [26].

\section{ОСОБЕННОСТИ ДИАГНОСТИКИ РАКА ПРЕДСТАТЕЛЬНОЙ ЖЕЛЕЗЫ У БОЛЬНЫХ С ОЖИРЕНИЕМ}

Многочисленные факты свидетельствуют о сложности обнаружения РПЖ у пациентов с избыточной массой тела. Во-первых, мужчины с ожирением имеют более низкие значения ПСА, что приводит к снижению частоты биопсии предстательной железы [27].

у пациентов с РПЖ и избыточным весом (ИМТ 25-30 кг/м²) значение ПСА ниже на 7\%, с ожирением (ИМТ 30-35 кг/м²) значение ПСА ниже на 14\%, у пациентов с тяжелым ожирением (ИМТ >35 кг/м²) значение ПСА ниже на $18 \%$ по сравнению с пациентами с нормаль-

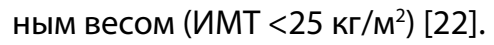

Аналогичное снижение уровня ПСА зарегистрировано для мужчин с избыточным весом и без ожирения [27]. Предполагается, что это связано с большим объемом плазмы у мужчин с ожирением, разбавляющим фиксированное количество ПСА (эффект гемодилюции) [28].

Во-вторых, ожирение усложняет проведение пальцевого ректального исследования (ПРИ), что приводит к еще большему количеству «поздних» диагнозов [29].

В-третьих, у мужчин с РПЖ и страдающих избыточной массой тела, снижается вероятность обнаружения рака при биопсии [30]. Более низкие значения ПСА в сочетании с трудностями при проведении ПРИ приводят к снижению количества положительных биопсий среди пациентов с избыточной массой тела. Большой объем предстательной железы может привести к большему количеству пропущенных опухолей, что в совокупности приводит к увеличению количества недиагностированных случаев РПЖ у пациентов с ожирением (рис. 1). В исследовании Rodriguez C., и его коллег даже в период до эры ПСА, при ранней биопсии ожирение было связано с РПЖ (в частности, со смертностью от данного заболевания) [31], и, таким образом, диагностическое «смещение» при обследовании (большая распространенность РПЖ у пациентов с ожирением) не может полностью объяснить связь между ожирением и агрессивным РПЖ.

\section{ВЛИЯНИЕ ОЖИРЕНИЯ НА КАНЦЕРСПЕЦИФИЧЕСКУЮ СМЕРТНОСТЬ ПРИ РАКЕ ПРЕДСТАТЕЛЬНОЙ ЖЕЛЕЗЫ}

Многочисленные большие когортные исследования последовательно продемонстрировали положительную корреляционную связь между увеличением ИМТ и летальным исходом при РПЖ [11, 20]. Метаанализ проспективных когортных исследований, включающих почти 7000 смертей, специфичных для РПЖ, показал 15-процентное увеличение смертности отданного заболевания при увеличении на 5 кг/м² увеличения ИМТ (ОР 1,15; 95\% ДИ 1,06-1,25) [32]. В метаанализе исследований типа «случай-контроль», включающих почти 1000 смертей, специфичных для РПЖ, сообщается о 20\% увеличении смертности, канцерспецифической для РПЖ при увеличении ИМТ на 5 кг/м² 


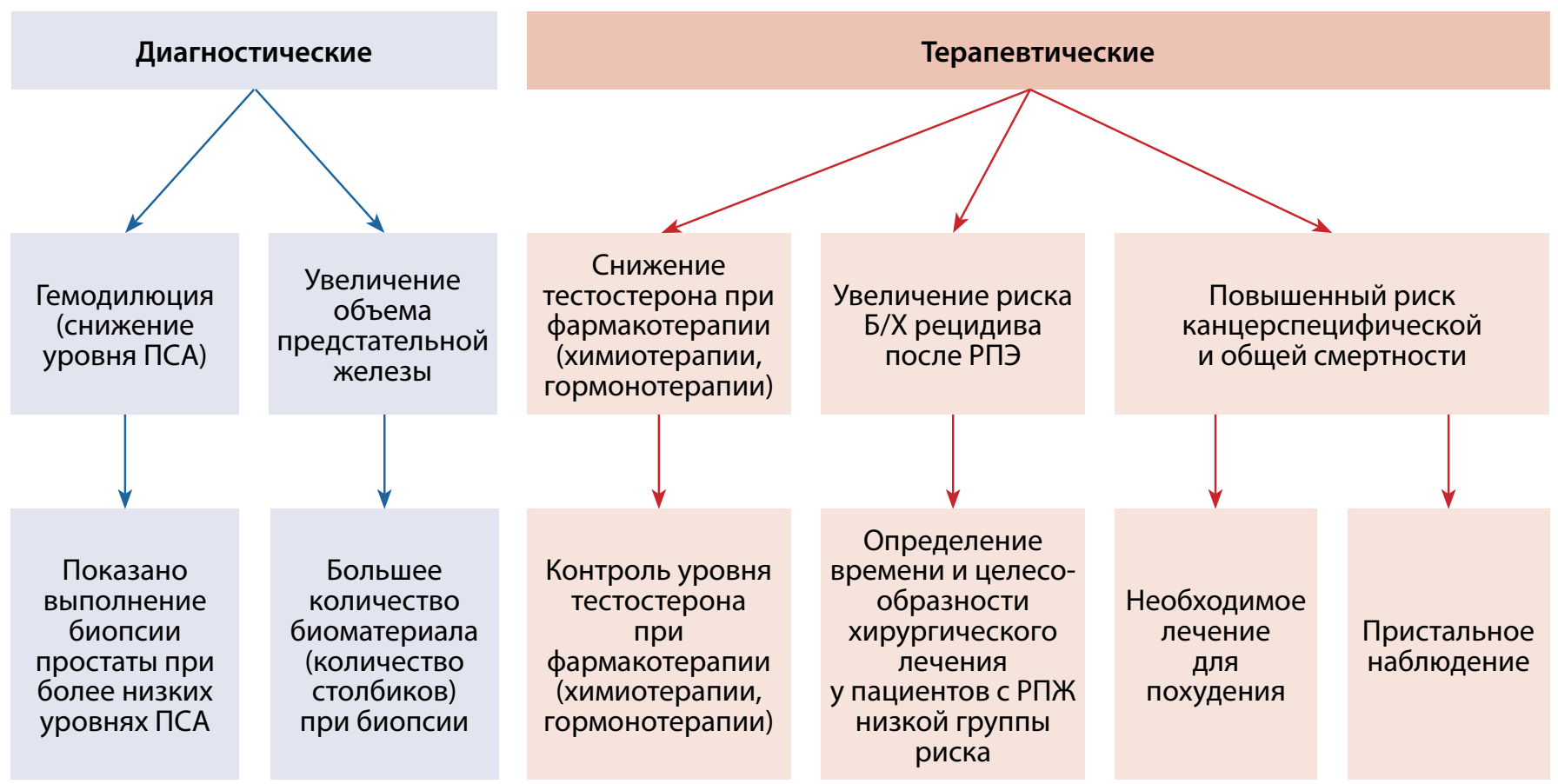

Рисунок 1. Диаграмма особенностей диагностики и терапии пациентов с раком предстательной железы с ожирением.

Примечание. АДТ - андроген-депривационная терапия; ПСА - простатспецифический антиген; РПЭ - радикальная простатэктомия; Б/X - биохимический.

(OP 1,20; 95\% ДИ 0,99-1,46) [32]. Сопоставимая величина OP, рассчитанная с применением большой популяционной выборки с различными схемами исследования, указывает на устойчивую связь между ожирением и смертностью от РПЖ. Данный эффект может быть частично обусловлен задержкой диагностики РПЖ у мужчин с ожирением по сравнению с мужчинами с нормальной массой тела. Ожирение связано со смертностью от РПЖ в период до применения ПСА-теста [20,31], это свидетельствует, что данная связь не может быть объяснена одной лишь особенностью выявления и что основные биологические механизмы играют важную роль.

Хотя связь между ожирением и смертностью от РПЖ по демографическим данным представляется весьма устойчивой, следует отметить, что за последние 20 лет отмечается увеличение числа лиц, страдающих ожирением, а смертность от РПЖ снижается [33]. Большая часть данного снижения смертности объясняется введением скрининга ПСА и улучшением результатов лечения, но это далеко не полное объяснение снижения смертности. Fesinmeyer M.D. и его коллеги, применяя имитационные модели, предположили, что если бы показатели ожирения в Соединенных Штатах оставались стабильными с 1980 г., уровень смертности от рака простаты в 2002 г. в Соединенных Штатах (последний год, за который у них были данные) был бы на 23\% ниже [34].

\section{МЕХАНИЗМЫ ВЛИЯНИЯ ОЖИРЕНИЯ НА РАК ПРЕДСТАТЕЛЬНОЙ ЖЕЛЕЗЫ}

Для объяснения связи между ожирением и агрессивным РПЖ чаще всего предлагаются три механизма:
1 - инсулиноподобный фактор роста (IGF)-1 [35];

2 - половые гормоны;

3 - цитокины и адипокины жировой ткани [36].

Концентрируемся на этих факторах, хотя следует признать, что существует множество других эффектов ожирения (воспаление, вызванное жирными кислотами, окислительный стресс, стресс эндоплазматического ретикулума и гипоксия) для биологии РПЖ [37].

\section{Инсулиноподобный фактор роста (IGF-1)}

Уровень циркулирующего инсулина положительно коррелирует с увеличением индекса массы тела, и пациенты, страдающие ожирением, обладают инсулинорезистентностью. Ожирение и длительная гиперинсулинемия связаны с повышением уровня свободного или биологически активного IGF-1 из-за снижения выработки IGF-связывающих белков, которые ингибируют его действие посредством взаимодействия с рецептором IGF. IGF активирует последующие сигнальные пути, которые влияют на рост опухолевых клеток путем стимулирования митогенных путей, индукции неоваскуляризации и ингибирования апоптоза. Инсулиноподобный фактор роста (IGF-1) обладает анаболическим, антиапоптотическим и митотическим эффектами [38].

Эпидемиологические данные свидетельствуют о том, что высокий уровень циркулирующего IGF-1 связан с повышенным риском развития РПЖ [39]. Передача сигналов IGF-1 повышена при РПЖ по сравнению с эпителием простаты и связана с прогрессированием опухоли [40]. В исследовании Hellawell G.O. и его коллег показана гиперэкспрессия рецептора IGF-1 при РПЖ [41]. 


\section{Половые гормоны}

Помимо изменений уровня инсулина, избыточная масса тела связана со снижением уровня андрогенов [42]. Суммарная андрогенная активность играет важную роль в росте и развитии железистого эпителия простаты, влияет на риск возникновения РПЖ.

Взаимосвязь между РПЖ и тестостероном впервые описана Хаггинсом более 70 лет назад [43] и носит противоречивый характер по мере накопления доказательств, демонстрирующих потенциально противоположное действие андрогенов на рак $[44,45,46]$. Тестостерон жизненно важен для нормального развития и роста ткани предстательной железы, и, наоборот, андрогенная депривационная терапия при метастатическом РПЖ значительно уменьшает симптомы и прогрессирование заболевания. На этом основании высокий уровень тестостерона увеличивает риск развития РПЖ. Однако эпидемиологические исследования не смогли продемонстрировать данную связь [47]. Моргенталер А. и соавт. [48], сообщили о высокой распространенности РПЖ среди бессимптомных форм у пациентов с низким уровнем общего и свободного тестостерона. Данное исследование является первым, показавшим, что низкий уровень тестостерона не обеспечивает защиту от развития РПЖ.

Также было обращено внимание на влияние тестостерона на гистологическую степень РПЖ. В ряде исследований было высказано предположение, что низкий уровень общего тестостерона в сыворотке крови может быть связан с заболеванием более высокой степени. Park et al. [49] ретроспективно оценили 681 пациента, перенесших биопсию простаты, и обнаружили независимую связь между лабораторным гипогонадизмом, определяемым как уровень общего тестостерона ниже 300 нг/дл, и высоким уровнем РПЖ при биопсии. Исследования, проведенные на пациентах с РПЖ с клинически локализованным заболеванием, получавшим радикальную простатэктомию (РП), также показали, что низкие уровни тестостерона в предоперационном периоде были связаны с более агрессивным заболеванием, обусловленным более высокой патологической стадией, положительными хирургическими краями и баллом по Глисону (GS) $[50,51]$. Тем не менее независимая связь не была одинаково продемонстрирована во всех исследованиях $[52,53]$. Большинство исследований были выполнены в популяциях Кавказа и Азии, но без указания влияния демографических характеристик на связь между уровнем тестостерона крови и РПЖ более высокого уровня. Возможные объяснения несоответствия между существующими исследованиями включают демографическую изменчивость, переменные ограничения для гипогонадизма и предвзятые выборки.

Таким образом, популяция пациентов с низким уровнем тестостерона и страдающих ожирением может быть одной из вероятных причин взаимосвязи между ожирением и агрессивным типом РПЖ, хотя точные механизмы остаются не до конца изученными [55].

Необходимо отметить, что тестостерон ароматизируется до эстрадиола в адипоцитах, клетках печени, предстательной железы, кожи и других органов [56]. Большая масса жировой ткани у пациентов с избыточным весом связана с активацией пути ароматизации, который приводит к повышению уровня эстрадиола в сыворотке и внутриклеточной ткани [54]. Хотя современные эпидемиологические данные не подтверждают связь между сывороточным эстрадиолом и риском развития РПЖ [57], значительный объем литературы по доклиническим исследованиям предполагает, что эстрадиол может содействовать развитию и прогрессированию РПЖ [58]. Таким образом, значение повышенных уровней эстрадиола у мужчин с ожирением в отношении риска и прогрессирования РПЖ еще предстоит определить.

Цитокины и адипокины жировой ткани

При ожирении в жировую ткань проникает большое количество воспалительных клеток (например, макрофагов), это связано с системным воспалением и резистентностью к инсулину [59]. Воспаление вызывает генерацию активных форм кислорода, которые действуют в качестве промоторов опухоли при низких концентрациях [60]. Адипоциты, другие стромальные клетки и инфильтрирующие воспалительные клетки в жировой ткани выделяют несколько адипокинов и других цитокинов, которые играют ключевую роль в развитии рака, связанного с ожирением [36]. Адипокины определяются как гормоноподобные полипептиды, которые активно секретируются белой жировой тканью, и они включают цитокины (например, интерлейкин-6 (IL-6) и фактор некроза опухоли (TNF-a)), ангиогенные факторы (например, фактор роста эндотелия сосудов (VEGF), апелин (AGTRL1)) и другие факторы (например, лептин и адипонектин) [61]. Было обнаружено, что несколько адипокинов оказывают множественное влияние на клетки рака предстательной железы.

\section{Лептин}

Лептин, полипептидный гормон, который в основном вырабатывается адипоцитами, действует в качестве основного регулятора аппетита и энергетического гомеостаза, воздействуя на специфические рецепторы, экспрессирующиеся в гипоталамусе [62]. Уровень лептина в плазме коррелирует с процентным содержанием жира в организме [63]. Это наблюдение предполагает, что большинство людей с ожирением нечувствительны к выработке эндогенного лептина.

В ткани предстательной железы человека рецепторы лептина обнаружены в нормальном эпителии, при интраэпителиальной неоплазии высокой степени и при аденокарциноме методом иммуногистохимического исследования [64]. Культуры клеток РПЖ (DU-145 и PC-3) экспрессируют рецепторы лептина. Воздействие лептина приводит к митогенному и антиапоптотическому эффекту этих клеток, активации пути фосфатидилинозитол-3-киназы (PI3К) и митоген-активируемой протеинкиназы (МАРК). Более того, длительное воздействие лептина усиливало пролиферацию, инвазию и миграцию клеток РПЖ с повышенной экспрессией циклина D1 и сниженной экспрессией р21, что свидетельствует об участии лептина в прогрессировании клеточного цикла [65]. Однонуклеотидный полиморфизм (SNP) в экзоне 4 гена рецептора лептина был достоверно связан со специфической смертностью от рака простаты [66].

Эпидемиологически связь между сывороточными уровнями лептина и риском развития рака простаты является спорной. Stattin P., и его коллеги сообщили, что 
умеренное повышение концентрации лептина в плазме связано с более поздним развитием РПЖ [64]. Исследование Li H. и его коллег показало, что повышенные концентрации лептина в плазме связаны с повышенным риском возникновения рака простаты большого объема (опухоль объемом >0,5 см) или с гистологическими признаками экстрапростатической инвазии, но без признаков метастазирования [67].

\section{IL-6}

Циркулирующий сывороточный IL-6 приводит к развитию инсулинорезистентности при ожирении [68]. В жировой ткани основными источниками IL-6 являются инфильтрирующие макрофаги и стромальные клетки. Однако адипоциты также продуцируют IL-6 [69]. Уровни сывороточного IL-6 были значительно выше у пациентов с метастатическим или гормонорефрактерным раком простаты, чем у пациентов контрольной группы или у пациентов с локализованным заболеванием, и они были связаны с более коротким временем выживания у пациентов с гормонорефрактерным раком простаты [70]. Экзогенный IL-6 стимулировал рост в гормоночувствительных клетках РПЖ LNCaP, но не гормоночувствительных клеточных линиях DU145 и PC-3. На модели ксенотрансплантата опухоли, когда клетки LNCaP, постоянно обрабатываемые IL-6, вводили голым мышам, объемы опухоли были больше, чем в группе контроля - опухолях, культивированных без обработки IL-6 [71]. Повышенная экспрессия рецептора IL-6 наблюдалась в ткани РПЖ человека по сравнению с нормальной тканью предстательной железы, а высокие уровни экспрессии рецептора IL-6 при РПЖы были связаны с более высокими показателями пролиферации клеток при иммуногистохимии [72].

\section{Адипонектин}

Адипонектин представляет собой белок, проявляющий структурное сходство с коллагеном и TNF-a, который в основном секретируется адипоцитами [73] и регулирует метаболизм глюкозы и липидов, ремоделирование сосудов и гомеостаз костей. Кроме того, адипонектин оказывает защитное действие против воспаления и резистентности к инсулину [74]. У людей концентрация адипонектина в плазме значительно ниже при ожирении и резистентности к инсулину. Адипонектин может связываться с тремя рецепторами, AdipoR1, AdipoR2 и T-cadherin [75]. Благодаря взаимодействию с AdipoR1 и AdipoR2, адипонектин проявляет антидиабетический эффект [76].

Уровень экспрессии AdipoR2 в клетках РПЖ положительно связан с пролиферацией клеток, с экспрессией синтазы жирных кислот и ангиогенезом [77]. Эти результаты предполагают положительную связь между AdipoR2 и развитием РПЖ.

Эпидемиологические исследования показали, что уровни адипонектина в плазме были значительно ниже у пациентов с РПЖ, чем у пациентов с доброкачественной гиперплазией предстательной железы (ДГПЖ) или у здоровых людей контрольной группы [78]. Tan W., и его коллеги изучали иммуногистохимические проявления адипонектина в случаях ДГПЖ и РПЖ с низким баллом по Глисону $(<7)$, баллом по шкале Глисона ( 7) или с высоким баллом по Глисону (>7). Результаты показали, что снижение экспрессии адипонектина связано с прогрессированием РПЖ [79].

\section{КАНЦЕРОГЕННАЯ МИКРОЭКОЛОГИЯ ЖИРОВОЙ ТКАНИ}

Системные эффекты, обусловленные ожирением, влияют на развитие РПЖ. Необходимо учитывать влияние микросреды, которая является основной детерминантой, определяющей рост локализованного рака, инвазию и метастазирование [80, 81].

Клинические исследования, оценивающие прогностическую ценность перипростатической жировой ткани, сообщают об ее увеличении при ожирении; является фактором риска как для диагностики рака простаты, так и для идентификации группы высокого прогностического риска [82].

В опухолевом микроокружении - перипростатической жировой ткани адипокины играют ключевую роль, действуя паракринно, аутокринно и эндокринно [83].

В исследовании Fain J.N. и соавт. сообщили, что уровни VEGF и IL-6, высвобождаемые из висцеральной жировой ткани, были значительно выше, чем из подкожной жировой ткани [84].

Таким образом, в случаях экстрапростатического распространения опухолевые клетки подвергаются непосредственному воздействию огромного количества адипокинов, высвобождаемых из перипростатической жировой ткани. Кроме того, увеличение перипростатической жировой ткани из-за ожирения изменяет микроокружение опухоли и ускоряет прогрессирование. Фактически, увеличение толщины перипростатической жировой ткани, измеренной с помощью трансректальной ультрасонографии как расстояния между простатой и лобковой костью, ассоциируется с раком простаты высокого прогностического риска [82].

Другим важным выводом является то, что пролиферация, вызванная микросредой перипростатической жировой ткани, была значительно увеличена по сравнению с аналогичной средой из подкожной или висцеральной жировой ткани $[85,86]$, что указывает на то, что перипростатическая жировая ткань секретирует уникальную подгруппу молекул, которая способствует агрессивности опухоли. Состав данного секретома остается не до конца изученным. Данные работы показывают, что перипростатическая жировая ткань секретирует факторы, которые способны изменять микроокружение ткани предстательной железы, способствуя пролиферации, выживанию и подвижности опухолевых клеток, и что этот секретом является более онкогенным у пациентов с ожирением.

\section{ЗАКЛЮЧЕНИЕ}

Результаты клинических исследований убедительно показывают влияние жировой ткани на малигнизацию ткани предстательной железы, включая индукцию опухолевого роста, инвазию и метастазирование. Опухолевые клетки предстательной железы при экстрапростатическом распространении образуют новое микроокружение в перипростатической жировой ткани. Считается, что опухолевые клетки напрямую и/или косвенно взаимодействуют с адипоцитами. 
Молекулярно-генетические механизмы влияния ожирения на развитие и прогрессирование опухоли недостаточно изучены на современном этапе. Сложно определить, какая часть результата связана с ожирением, видом проводимой терапии или молекулярно-биологическим типом опухоли. Адипокины, по-видимому, являются ключевыми молекулами в отношениях между опухолевыми клетками и жировой тканью. При гиперплазии и гипертрофии жировой ткани выделяется ряд адипокинов, которые могут играть роль в развитии опухоли. Адипоциты, выделяя воспалительные цитокины, фактор некроза опухоли-а (TNF-a) и интерлейкин-6 (ИЛ-6), вызывают хроническую воспалительную микросреду, которая также способствует прогрессированию опухолевого роста. Жировая ткань выделяет лептин и адипонектин, которые связаны с РПЖ. Таким образом, жировая ткань влияет на биологический фенотип опухоли, способствует проявлению агрессивных свойств.
Более глубокое понимание роли жировой ткани в прогрессировании РПЖ приведет к более эффективным терапевтическим стратегиям и улучшит результаты лечения при данном заболевании.

\section{ДОПОЛНИТЕЛЬНАЯ ИНФОРМАЦИЯ}

Источник финансирования. Подготовка и публикация рукописи проведены на личные средства авторского коллектива.

Конфликт интересов. Обзор является частью диссертационного исследования М.Н. Пешкова. Остальные авторы декларируют отсутствие явных и потенциальных конфликтов интересов, связанных с публикацией настоящей статьи.

Участие авторов: Пешков М.Н. - сбор, анализ данных литературы, написание статьи; Пешкова Г.П. - редактирование статьи; Решетов И.В. - редактирование статьи. Все авторы внесли значимый вклад в проведение исследования и подготовку статьи, прочли и одобрили финальную версию статьи перед публикацией.

\section{СПИСОК ЛИТЕРАТУРЫ | REFERENCES}

1. Злокачественные новообразования в России в 2017 году (заболеваемость и смертность). / Под ред. Каприна А.Д. Старинского В.В., Петровой Г.В. - М.: МНИОИ им. П.А. Герцена филиал ФГБУ «НМИЦ радиологии» Минздрава России; 2018 [Kaprin AD, Starinskiy V, Petrova GV, editors. Zlokachestvennye novoobrazovaniya $v$ Rossii v 2017 godu (zabolevaemost' i smertnost'). Moscow: MNIOI im. P.A. Gertsena - filial FGBU «NMITs radiologii» Minzdrava Rossii; 2018. (In Russ.)]

2. Seer.cancer.gov [Internet]. Nacional Cancer Institute [cited 2020 Aug 19]. Available from: https://seer.cancer.gov.

3. Altekruse SF, Kosary CL, Krapcho M, et al. SEER Cancer Statistics Review, 1975-2007. Bethesda: National Cancer Institute; 2010.

4. Kitagawa Y, Namiki M. Prostate-specific antigen-based population screening for prostate cancer: current status in Japan and future perspective in Asia. Asian J Androl. 2015;17(3):475-480. doi: https://doi.org/10.4103/1008-682X.143756

5. Swindle P, Eastham JA, Ohori M, et al. Do margins matter? The prognostic significance of positive surgical margins in radical prostatectomy specimens. J Urol. 2005;174(3):903-907. doi: https://doi.org/10.1097/01.ju.0000169475.00949.78

6. Sung MT, Eble JN, Cheng L. Invasion of fat justifies assignment of stage pT3a in prostatic adenocarcinoma. Pathology. 2006;38(4):309-311. doi: https://doi.org/10.1080/00313020600820914

7. Kyrgiou M, Kalliala I, Markozannes G, et al. Adiposity and cancer at major anatomical sites: umbrella review of the literature. BMJ. 2017;356:j477. doi: https://doi.org/10.1136/bmj.j477

8. Дедов И.И., Мельниченко Г.А., Шестакова М.В., и др. Национальные клинические рекомендации по лечению морбидного ожирения у взрослых. 3-ий пересмотр (лечение морбидного ожирения у взрослых). // Ожирение и метаболизм. - 2018. - Т. 15. №1. - C. 53-70. [Dedov II, Mel'nichenko GA, Shestakova MV, et al. Russian national clinical recommendations for morbid obesity treatment in adults. 3rd revision (Morbid obesity treatment in adults). Obesity and metabolism. 2018;15(1):53-70. (In Russ.)] doi: https://doi.org/10.14341/omet2018153-70

9. Gilbert CA, Slingerland JM. Cytokines, obesity, and cancer: new insights on mechanisms linking obesity to cancer risk and progression. Annu Rev Med. 2013;64:45-57 doi: https://doi.org/10.1146/annurev-med-121211-091527

10. Wang $Y Y$, Lehuede $C$, Laurent $V$, et al. Adipose tissue and breast epithelial cells: a dangerous dynamic duo in breast cancer. Cancer Lett. 2012;324(2):142-151. doi: https://doi.org/10.1016/j.canlet.2012.05.019

11. Calle EE, Rodriguez C, Walker-Thurmond K, Thun MJ. Overweight, obesity, and mortality from cancer in a prospectively studied cohort of U.S. adults. N Engl J Med. 2003;348(17):1625-1638. doi: https://doi.org/10.1056/NEJMoa021423

12. Allott EH, Masko EM, Freedland SJ. Obesity and prostate cancer: weighing the evidence. Eur Urol. 2013;63(5):800-809. doi: https://doi.org/10.1016/j.eururo.2012.11.013
13. Vidal AC, Howard LE, Moreira DM, et al. Obesity increases the risk for high-grade prostate cancer: results from the REDUCE study. Cancer Epidemiol Biomarkers Prev. 2014;23(12):2936-2942. doi: https://doi.org/10.1158/1055-9965.EPI-14-0795

14. Rundle A, Jankowski M, Kryvenko ON, et al. Obesity and future prostate cancer risk among men after an initial benign biopsy of the prostate. Cancer Epidemiol Biomarkers Prev. 2013;22(5):898-904 doi: https://doi.org/10.1158/1055-9965.EPI-12-0965

15. Renehan AG, Tyson M, Egger M, et al. Body-mass index and incidence of cancer: a systematic review and meta-analysis of prospective observational studies. Lancet. 2008;371(9612):569-578. doi: https://doi.org/10.1016/s0140-6736(08)60269-x

16. Maclnnis RJ, English DR. Body size and composition and prostate cancer risk: systematic review and meta-regression analysis. Cancer Causes Control. 2006;17(8):989-1003. doi: https://doi.org/10.1007/s10552-006-0049-z

17. Bergström A, Pisani $P$, Tenet $V$, et al. Overweight as an avoidable cause of cancer in Europe. Int J Cancer. 2001;91(3):421-430. doi: https://doi. org/10.1002/1097-0215(200002)9999:9999<::aid-ijc1053>3.0.co;2-t

18. Baillargeon J, Platz EA, Rose DP, et al. Obesity, adipokines, and prostate cancer in a prospective population-based study. Cancer Epidemiol Biomarkers Prev. 2006;15(7):1331-1335. doi: https://doi.org/10.1158/1055-9965.EPI-06-0082

19. Engeland A, Tretli S, Bjorge T. Height, body mass index, and prostate cancer: a follow-up of 950000 Norwegian men. Br J Cancer. 2003;89(7):1237-1242. doi: https://doi.org/10.1038/sj.bjc.6601206

20. Andersson SO, Wolk A, Bergstrom R, et al. Body size and prostate cancer: a 20-year follow-up study among 135006 Swedish construction workers. J Nat/ Cancer Inst. 1997;89(5):385-389. doi: https://doi.org/10.1093/jnci/89.5.385

21. Bergström $A$, Pisani $P$, Tenet $V$, et al. Overweight as an avoidable cause of cancer in Europe. Int J Cancer. 2001;91(3):421-430. doi: https://doi. org/10.1002/1097-0215(200002)9999:9999<::aid-ijc1053>3.0.co;2-t

22. Banez LL, Hamilton RJ, Partin AW, et al. Obesity-related plasma hemodilution and PSA concentration among men with prostate cancer. JAMA. 2007;298(19):2275-2280. doi: https://doi.org/10.1001/jama.298.19.2275

23. Gong Z, Neuhouser ML, Goodman PJ, et al. Obesity, diabetes, and risk of prostate cancer: results from the prostate cancer prevention trial. Cancer Epidemiol Biomarkers Prev. 2006;15(10):1977-1983. doi: https://doi.org/10.1158/1055-9965.EPI-06-0477

24. Wright ME, Chang SC, Schatzkin A, et al. Prospective study of adiposity and weight change in relation to prostate cancer incidence and mortality. Cancer. 2007;109(4):675-684 doi: https://doi.org/10.1002/cncr.22443

25. Burton A, Martin R, Galobardes B, et al. Young adulthood body mass index and risk of cancer in later adulthood: historical cohort study. Cancer Causes Control. 2010;21(12):2069-2077. doi: https://doi.org/10.1007/s10552-010-9625-3 
26. Okasha M, McCarron P, McEwen J, Smith GD. Body mass index in young adulthood and cancer mortality: a retrospective cohort study. J Epidemiol Community Health. 2002;56(10):780-784. doi: https://doi.org/10.1136/jech.56.10.780

27. Hekal IA, Ibrahiem El. Obesity-PSA relationship: a new formula. Prostate Cancer Prostatic Dis. 2010;13(2):186-190. doi: https://doi.org/10.1038/pcan.2009.53

28. Grubb RL, 3rd, Black A, Izmirlian G, et al. Serum prostate-specific antigen hemodilution among obese men undergoing screening in the Prostate, Lung, Colorectal, and Ovarian Cancer Screening Trial. Cancer Epidemiol Biomarkers Prev. 2009;18(3):748-751. doi: https://doi.org/10.1158/1055-9965.EPI-08-0938

29. Hu MB, Liu SH, Jiang HW, et al. Obesity affects the biopsy-mediated detection of prostate cancer, particularly high-grade prostate cancer: a dose-response meta-analysis of 29,464 patients. PLoS One. 2014:9(9):e106677. doi: https://doi.org/10.1371/journal.pone.0106677

30. Freedland SJ, Platz EA, Presti JC, et al. Obesity, Serum Prostate Specific Antigen and Prostate Size: Implications for Prostate Cancer Detection. J Urol. 2006;175(2):500-504. doi: https://doi.org/10.1016/s0022-5347(05)00162-x

31. Rodriguez C, Patel AV, Calle EE, et al. Body mass index, height, and prostate cancer mortality in two large cohorts of adult men in the United States. Cancer Epidemiol Biomarkers Prev. 2001:10(4):345-353.

32. Cao Y, Ma J. Body mass index, prostate cancer-specific mortality, and biochemical recurrence: a systematic review and meta-analysis. Cancer Prev Res (Phila). 2011;4(4):486-501 doi: https://doi.org/10.1158/1940-6207.CAPR-10-0229

33. Collin SM, Martin RM, Metcalfe C, et al. Prostate-cancer mortality in the USA and UK in 1975-2004: an ecological study. Lancet Oncol. 2008;9(5):445-452. doi: https://doi.org/10.1016/s1470-2045(08)70104-9

34. Fesinmeyer MD, Gulati R, Zeliadt S, et al. Effect of Population Trends in Body Mass Index on Prostate Cancer Incidence and Mortality in the United States. Cancer Epidemiol Biomarkers Prev. 2009:18(3):808-815. doi: https://doi.org/10.1158/1055-9965.epi-08-0784

35. Renehan AG, Frystyk J, Flyvbjerg A. Obesity and cancer risk: the role of the insulin-IGF axis. Trends Endocrinol Metab. 2006;17(8):328-336. doi: https://doi.org/10.1016/j.tem.2006.08.006

36. Roberts DL, Dive C, Renehan AG. Biological mechanisms linking obesity and cancer risk: new perspectives. Annu Rev Med. 2010;61:301-316. doi: https://doi.org/10.1146/annurev.med.080708.082713

37. Sharad S, Srivastava A, Ravulapalli S, et al. Prostate cancer gene expression signature of patients with high body mass index. Prostate Cancer Prostatic Dis. 2011;14(1):22-29. doi: https://doi.org/10.1038/pcan.2010.44

38. De Pergola G, Silvestris F. Obesity as a major risk factor for cancer. J Obes. 2013;2013:291546. doi: https://doi.org/10.1155/2013/291546

39. Roddam AW, Allen NE, Appleby P, et al. Insulin-like growth factors, their binding proteins, and prostate cancer risk: analysis of individual patient data from 12 prospective studies. Ann Intern Med. 2008;149(7):461-471, W483-468. doi: https://doi.org/10.7326/0003-4819-149-7-200810070-00006

40. KrueckI SL, Sikes RA, Edlund NM, et al. Increased insulin-like growth factor I receptor expression and signaling are components of androgen-independent progression in a lineage-derived prostate cancer progression model. Cancer Res. 2004;64(23):8620-8629. doi: https://doi.org/10.1158/0008-5472.CAN-04-2446

41. Hellawell GO, Turner GD, Davies DR, et al. Expression of the type 1 insulin-like growth factor receptor is up - regulated in primary prostate cancer and commonly persists in metastatic disease. Cancer Res. 2002:62(10):2942-2950.

42. Williams G. Aromatase up-regulation, insulin and raised intracellular oestrogens in men, induce adiposity, metabolic syndrome and prostate disease, via aberrant ER-alpha and GPER signalling. Mol Cell Endocrinol. 2012;351(2):269-278. doi: https://doi.org/10.1016/j.mce.2011.12.017

43. Huggins C. Studies on Prostatic Cancer. Arch Surg. 1941;43(2):209. doi: https://doi.org/10.1001/archsurg.1941.01210140043004

44. Morgentaler A. Testosterone replacement therapy and prostate cancer. Urol Clin North Am. 2007;34(4):555-563, vii. doi: https://doi.org/10.1016/j.ucl.2007.08.002

45. Roddam AW, et al. Endogenous sex hormones and prostate cancer: a collaborative analysis of 18 prospective studies. J Nat/ Cancer Inst. 2008;100(3):170-183. doi: https://doi.org/10.1093/jnci/djm323
46. Isbarn $\mathrm{H}$, Pinthus JH, Marks LS, et al. Testosterone and prostate cancer: revisiting old paradigms. Eur Urol. 2009;56(1):48-56. doi: https://doi.org/10.1016/j.eururo.2009.03.088

47. Morgentaler A. Turning conventional wisdom upside-down: low serum testosterone and high-risk prostate cancer. Cancer. 2011;117(17):3885-3888. doi: https://doi.org/10.1002/cncr.25975

48. Morgentaler A. Occult Prostate Cancer in Men With Low Serum Testosterone Levels. JAMA. 1996;276(23):1904. doi: https://doi.org/10.1001/jama.1996.03540230054035

49. Park J, Cho SY, Jeong S-h, et al. Low testosterone level is an independent risk factor for high-grade prostate cancer detection at biopsy. BJU Int. 2016;118(2):230-235. doi: https://doi.org/10.1111/bju.13206

50. Isom-Batz G, Bianco FJ, Jr., Kattan MW, et al. Testosterone as a predictor of pathological stage in clinically localized prostate cancer. J Urol. 2005;173(6):1935-1937. doi: https://doi.org/10.1097/01.ju.0000158040.33531.e7

51. Imamoto T, Suzuki H, Fukasawa S, et al. Pretreatment serum testosterone level as a predictive factor of pathological stage in localized prostate cancer patients treated with radical prostatectomy. Eur Urol. 2005;47(3):308-312. doi: https://doi.org/10.1016/j.eururo.2004.11.003

52. Salonia A, Gallina A, Briganti A, et al. Preoperative hypogonadism is not an independent predictor of high-risk disease in patients undergoing radical prostatectomy. Cancer. 2011;117(17):3953-3962. doi: https://doi.org/10.1002/cncr.25985

53. Shin BS, Hwang EC, Im CM, et al. Is a decreased serum testosterone level a risk factor for prostate cancer? A cohor study of korean men. Korean J Urol. 2010;51(12):819-823. doi: https://doi.org/10.4111/kju.2010.51.12.819

54. Theoret MR, Ning YM, Zhang JJ, et al. The risks and benefits of 5 alphareductase inhibitors for prostate-cancer prevention. N Engl J Med. 2011;365(2):97-99. doi: https://doi.org/10.1056/NEJMp1106783

55. Wang G, Zhao D, Spring DJ, DePinho RA. Genetics and biology of prostate cancer. Genes Dev. 2018:32(17-18):1105-1140. doi: https://doi.org/10.1101/gad.315739.118

56. Carruba G. Estrogen and prostate cancer: an eclipsed truth in an androgen-dominated scenario. J Cell Biochem. 2007:102(4):899-911. doi: https://doi.org/10.1002/jcb.21529

57. Yao S, Till C, Kristal AR, et al. Serum estrogen levels and prostate cancer risk in the prostate cancer prevention trial: a nested case-control study. Cancer Causes Control. 2011;22(8):1121-1131. doi: https://doi.org/10.1007/s10552-011-9787-7

58. Bonkhoff $\mathrm{H}$, Berges R. The Evolving Role of Oestrogens and Their Receptors in the Development and Progression of Prostate Cancer. Eur Urol. 2009:55(3):533-542. doi: https://doi.org/10.1016/j.eururo.2008.10.035

59. Weisberg SP, McCann D, Desai M, et al. Obesity is associated with macrophage accumulation in adipose tissue. J Clin Invest 2003;112(12):1796-1808. doi: https://doi.org/10.1172/JCI19246

60. Ziech D, Franco R, Pappa A, Panayiotidis MI. Reactive oxygen species (ROS)--induced genetic and epigenetic alterations in human carcinogenesis. Mutat Res. 2011;711(1-2):167-173. doi: https://doi.org/10.1016/j.mrfmmm.2011.02.015

61. Kershaw EE, Flier JS. Adipose tissue as an endocrine organ. J Clin Endocrinol Metab. 2004;89(6):2548-2556. doi: https://doi.org/10.1210/jc.2004-0395

62. Li MD. Leptin and beyond: an odyssey to the central control of body weight. Yale J Biol Med. 2011;84(1):1-7.

63. Considine RV, Sinha MK, Heiman ML, et al. Serum immunoreactive-leptin concentrations in normal-weight and obese humans. N Engl J Med. 1996;334(5):292-295. doi: https://doi.org/10.1056/NEJM199602013340503

64. Stattin P, Soderberg S, Hallmans G, et al. Leptin is associated with increased prostate cancer risk: a nested case-referent study. J Clin Endocrinol Metab. 2001;86(3):1341-1345. doi: https://doi.org/10.1210/jcem.86.3.7328

65. Noda T, Kikugawa T, Tanji N, et al. Longterm exposure to leptin enhances the growth of prostate cancer cells. Int J Oncol. 2015;46(4):1535-1542. doi: https://doi.org/10.3892/ijo.2015.2845

66. Lin DW, FitzGerald LM, Fu R, et al. Genetic variants in the LEPR, CRY1, RNASEL, IL4, and ARVCF genes are prognostic markers of prostate cancer-specific mortality. Cancer Epidemiol Biomarkers Prev. 2011;20(9):1928-1936. doi: https://doi.org/10.1158/1055-9965.EPI-11-0236 
67. Li H, Stampfer MJ, Mucci L, et al. A 25-year prospective study of plasma adiponectin and leptin concentrations and prostate cancer risk and survival. Clin Chem. 2010;56(1):34-43. doi: https://doi.org/10.1373/clinchem.2009.133272

68. Kern PA, Ranganathan S, Li C, et al. Adipose tissue tumor necrosis factor and interleukin-6 expression in human obesity and insulin resistance. Am J Physiol Endocrinol Metab. 2001;280(5):E745-751. doi: https://doi.org/10.1152/ajpendo.2001.280.5.E745

69. Fried SK, Bunkin DA, Greenberg AS. Omental and subcutaneous adipose tissues of obese subjects release interleukin-6: depot difference and regulation by glucocorticoid. J Clin Endocrinol Metab. 1998;83(3):847-850. doi: https://doi.org/10.1210/jcem.83.3.4660

70. George DJ, Halabi S, Shepard TF, et al. The prognostic significance of plasma interleukin-6 levels in patients with metastatic hormone-refractory prostate cancer: results from cancer and leukemia group B 9480. Clin Cancer Res. 2005;1 1(5):1815-1820. doi: https://doi.org/10.1158/1078-0432.CCR-04-1560

71. Steiner H, Godoy-Tundidor S, Rogatsch $\mathrm{H}$, et al. Accelerated in Vivo Growth of Prostate Tumors that Up-Regulate Interleukin-6 Is Associated with Reduced Retinoblastoma Protein Expression and Activation of the Mitogen-Activated Protein Kinase Pathway. Am J Pathol. 2003;162(2):655-663. doi: https://doi.org/10.1016/s0002-9440(10)63859-x

72. Giri D, Ozen M, Ittmann M. Interleukin-6 Is an Autocrine Growth Factor in Human Prostate Cancer. Am J Patho. 2001;159(6):2159-2165. doi: https://doi.org/10.1016/s0002-9440(10)63067-2

73. Wang Y, Xu A, Knight C, et al. Hydroxylation and Glycosylation of the Four Conserved Lysine Residues in the Collagenous Domain of Adiponectin. J Biol Chem. 2002;277(22):19521-19529. doi: https://doi.org/10.1074/jbc.M200601200

74. Tilg H, Moschen AR. Adipocytokines: mediators linking adipose tissue, inflammation and immunity. Nat Rev Immunol. 2006;6(10):772-783. doi: https://doi.org/10.1038/nri1937

75. Dalamaga M, Diakopoulos KN, Mantzoros CS. The role of adiponectin in cancer: a review of current evidence. Endocr Rev. 2012;33(4):547-594. doi: https://doi.org/10.1210/er.2011-1015

76. Kadowaki T, Yamauchi T, Kubota N, et al. Adiponectin and adiponectin receptors in insulin resistance, diabetes, and the metabolic syndrome. J Clin Invest. 2006;1 16(7):1784-1792. doi: https://doi.org/10.1172/JCI29126
77. Rider JR, Fiorentino M, Kelly $R$, et al. Tumor expression of adiponectin receptor 2 and lethal prostate cancer. Carcinogenesis. 2015;36(6):639-647. doi: https://doi.org/10.1093/carcin/bgv048

78. Michalakis K, Venihaki M, Mantzoros C, et al. In prostate cancer, low adiponectin levels are not associated with insulin resistance. Eur J Clin Invest. 2015;45(6):572-578. doi: https://doi.org/10.1111/eci.12445

79. Tan W, Wang L, Ma Q, et al. Adiponectin as a potential tumor suppressor inhibiting epithelial-to-mesenchymal transition but frequently silenced in prostate cancer by promoter methylation. Prostate. 2015;75(11):1197-1205. doi: https://doi.org/10.1002/pros.23002

80. Hanahan D, Weinberg RA. Hallmarks of cancer: the next generation. Cell. 2011;144(5):646-674 doi: https://doi.org/10.1016/j.cell.2011.02.013

81. Taylor RA, Risbridger GP. Prostatic tumor stroma: a key player in cancer progression. Curr Cancer Drug Targets. 2008;8(6):490-497. doi: https://doi.org/10.2174/156800908785699351

82. Bhindi B, Trottier G, Elharram M, et al. Measurement of periprostatic fat thickness using transrectal ultrasonography (TRUS): a new risk factor for prostate cancer. BJU Int. 2012;110(7):980-986. doi: https://doi.org/10.1111/j.1464-410X.2012.10957.x

83. Booth A, Magnuson A, Fouts J, Foster M. Adipose tissue, obesity and adipokines: role in cancer promotion. Horm Mol Biol Clin Investig. 2015;21(1):57-74. doi: https://doi.org/10.1515/hmbci-2014-0037

84. Fain JN, Madan AK, Hiler ML, et al. Comparison of the release of adipokines by adipose tissue, adipose tissue matrix, and adipocytes from visceral and subcutaneous abdominal adipose tissues of obese humans. Endocrinology. 2004;145(5):2273-2282 doi: https://doi.org/10.1210/en.2003-1336

85. Venkatasubramanian PN, Brendler CB, Plunkett BA, et al. Periprostatic adipose tissue from obese prostate cancer patients promotes tumor and endothelial cell proliferation: a functional and MR imaging pilot study. Prostate. 2014;74(3):326-335. doi: https://doi.org/10.1002/pros.22756

86. Ribeiro R, Monteiro C, Cunha V, et al. Human periprostatic adipose tissue promotes prostate cancer aggressiveness in vitro. J Exp Clin Cancer Res. 2012;31:32. doi: https://doi.org/10.1186/1756-9966-31-32

\section{ИНФОРМАЦИЯ ОБ АВТОРАХ [AUTHORS INFO]:}

*Пешков Максим Николаевич, к.м.н. [Maxim N. Peshkov, MD, PhD]; адрес: Россия, 125371, Москва, Волоколамское ш., д. 91 [address: 91, Volokolamskoye Highway street, 125371 Moscow, Russia]; ORCID: https://orcid.org/0000-0002-1497-8646; eLibrary SPIN: 1691-1478; e-mail: drpeshkov@gmail.com

Пешкова Галина Петровна, к.M.H. [Galina P. Peshkova, MD, PhD]; ORCID: https://orcid.org/0000-0002-3336-4025; eLibrary SPIN: 6849-5407; e-mail: gppeshkova@gmail.com Решетов Игорь Владимирович, Д.м.H. [Igor V. Reshetov, MD, PhD]; ORCID: https://orcid.org/0000-0002-5739-3170; eLibrary SPIN: 3845-6604; e-mail: reshetoviv@mail.ru

*Автор, ответственный за переписку / Corresponding author.

\section{ЦИТИРОВАТЬ:}

Пешков М.Н., Пешкова Г.П., Решетов И.В. Взаимосвязь ожирения и рака предстательной железы (обзор) // Ожирение и метаболизм. - 2020. - Т. 17. - №2. - С. 147-155. doi: https://doi.org/10.14341/omet10301

\section{TO CITE THIS ARTICLE:}

Peshkov MN, Peshkova GP, Reshetov IV. The relationship of obesity and prostate cancer (review). Obesity and metabolism. 2020;17(2):147-155. doi: https://doi.org/10.14341/omet10301 